\title{
Brachytherapy next generation: robotic systems
}

\author{
Tiberiu Popescu, MScl, Alex Cristian Kacsó, MScl, Prof. Doina Pisla, PhD², Assoc. Prof. Gabriel Kacsó, MD, PhD1.3 \\ I"Iuliu Haţieganu" University of Medicine and Pharmacy, Cluj-Napoca, ${ }^{2}$ Technical University of Cluj-Napoca, The Research Center for Industrial \\ Robots Simulation and Testing, Cluj, ${ }^{3}$ Department of Radiation Oncology and Medical Oncology, RTC Amethyst Cluj, Romania
}

\begin{abstract}
In a field dominated by external beam radiation therapy (EBRT), both the therapeutic and technical possibilities of brachytherapy (BT) are underrated, shadowed by protons and intensity modulated radiotherapy. Decreasing expertise and indications, as well as increasing lack of specific BT training for radiation therapy (RT) residents led to the real need of shortening its learning curve and making it more popular. Developing robotic BT devices can be a way to mitigate the above issues. There are many teams working at custom-made robotic BT platforms to perfect and overcome the limitations of the existing systems. This paper provides a picture of the current state-of-the-art in robotic assisted BT, as it also conveys the author's solution to the problem, a parallel robot that uses CT-guidance.
\end{abstract}

Key words: brachytherapy, image-guidance, parallel robots, robotic systems.

\section{Purpose}

For some, brachytherapy (BT) is something on the brink of extinction and their arguments are not scarce. Brachytherapy might not sound too appealing for the average radiation oncologist because it implies transition from behind the screen to the OR. Blood appears as a real scenario and most of them chose from the beginning to avoid it willingly. It is not only about the lack of surgical skills, but also that most junior radiation oncologists do not actually have the chance to see or practice the technique in their centers. Moreover, BT is by itself a niche domain, as there are rather few indications for it, as compared to external beam radiotherapy (EBRT). Therefore, for most of them, BT is something more like "see not touch". Last but not least, in a domain driven by EBRT, investing in BT might not seem too appealing for developers.

Fortunately, some of these issues can be corrected. With proper indications and good technique, BT can achieve excellent results. Thus, in their effort to keep the domain alive, doctors that advocate for it tried to replace the human hand for the "bloody" part while keeping the radiation therapy spirit friendly to radiation oncologists. As a result of this struggle for accessibility, safety, precision, and accuracy, BT robotic systems appeared.

The aim of this paper is to evaluate the current situation in the field of robotic brachytherapy, briefly outline the existing systems, their technical aspects, and limitations, and to present a custom-made solution to the problem, a universal brachytherapy robotic system, ParaBrachyRob.

\section{Technical considerations}

The purpose of BT is to achieve, by interstitial radioactive seed implantation (low-dose-rate - LDR brachytherapy) or temporary source implantation (high-dose-rate - HDR, or pulse-dose-rate - PDR brachytherapy), a high conformal dose distribution to cover the target volume. At the same time, BT allows a steep decrease of the dose outside of the desired volume. It is thus an efficient treatment for virtual any small to medium sized well defined tumor, which can be reached by visual/palpable guidance, provided that correct indications and accurate technique are met. While the indication is based only on knowledge, usually set by multi-disciplinary team (MDT) decision, the technique requires a high degree of skill and the use of real-time image guidance, which can be improved by automation. The report of the joint Task Group 192 of the American Association of Physicists in Medicine (AAPM) and the Groupe Européen de Curiethérapie (GEC)-European Society for Radiotherapy \& Oncology (ESTRO) reviews the Robotics Institute of America's (RIA) definition and classification of robots, and makes a clear distinction between "automation" and "autonomy" [1]. It raises two fundamental questions on "autonomy by an automatic system": autonomy from whom? and autonomy to do what? The first addresses the matter of permission for the robot to conduct its operations without human supervision. The second builds on the "degrees of automation" described by Sheridan (plan, teach, monitor, intervene, learn) [2] and refers to treatment execution, supervision of treatment planning, monitoring, and intervention. It also proposes the modi-

\footnotetext{
Address for correspondence: Assoc. Prof. Gabriel Kacsó, MD, PhD, RTC Amethyst, 486G Razoare, 
fication of system performance based on previous learning or teaching experiences.

Based on these degrees, the report proposes a new classification of automatic systems, more appropriate for BT robots and different from that elaborated by the RIA. Instead of subdividing robots into Classes (1-4), it puts them on four Levels of Autonomy (I-IV). The denomination "Class (1-4) robot operating at Level of Autonomy (I-IV)" might not be uncommon. There are currently no available Level IV medical robots, i.e. machines capable to create and complete all the tasks without human interaction [1].

As they yet do not possess full autonomy and work in a human environment, BT robotic systems are required to meet the SAUR (safety, accuracy, user-friendliness, and reliability) criteria. Indeed, apart from widening the indications of the technique, increasing its availability by automation and by reducing the learning curve, these robots aim at improving accuracy. When applied for permanent BT, this relates to precise seed placement, finding optimal seed locations, decreasing surgical trauma, and finally protecting medical staff from radiation exposure. An extensive list of the additional functional recommendations on robotic BT systems can be consulted in the joint Task Group 192's Report [1].

However, the ultimate purpose is not to bring a machine to the existing level of human expertise but to better implants and enhance the quality of care in a consistent, reproducible way. This means extending the indications of BT while keeping it at the same level of minimal invasiveness or even increasing the efficacy/toxicity ratio. A checklist that should be satisfied by every BT robotic system in this respect is provided by the same report. In terms of accuracy, manual seeds placement have an accuracy of 3-6 mm when placed in vivo with a rigid template. Conversely, robotic systems are required to acquire a spatial accuracy of less than $1 \mathrm{~mm}$ in phantom models. They are also demanded to solve issues such as tissue deformation (by rotational needle insertion), needle deviation (fixed needle trajectory, sensors for pressure and pulsations), and to avoid edema, which would prevent the correct distribution of the delivered dose as planned pre-implant and intra-operatively (dynamic planning by update of dosimetry). Patient position and existing imaging technology can be limiting the robotic system's degree of freedom (DOF), and construction materials (for example, the dorsal-lithotomic position for prostate brachytherapy might impair the transperineal insertion with direct visualization on CT and/or MRI because of the limited space in the CT/MRI/Bore; all the cables for MRI guided robot must be with no magnetizable material such iron or copper, rising significantly the costs). System adaptability is advised so that the future advances in 3D imaging technology could offer a real advantage to these systems. Prostate deformation/rotation during implant and bevel-tip needle steering effect lead also to problems such as significant target displacements and spinning, respectively. At the same time, reaching a higher level of autonomy is hindered by the operator's need of manual control and the consequent preference for a master-slave interface. From this perspective, the catheter placement in the abdomen, pelvis, or thorax can be guided by the da Vinci surgery robot, after resection, biopsy, or if the cancer is judged inoperable.

\section{Existing systems}

Image-guided brachytherapy (IGBT) comprises BT robotic systems that currently use MRI (magnetic resonance imaging), CT (computed tomography), or TRUS (trans-rectal ultrasound). While the first provides the best soft tissue contrast, the last is the most commonly used due to cost and availability advantages. There are a dozen of robotic systems developed worldwide, few to have been used on patients (FIRST [3], EUCLIDEAN [4], and JHU1 [5]). Only one has received the FDA and $\mathrm{CE}$ approvals and become commercially available for prostate BT applications: Fully Integrated Real-time Seed Treatment (FIRST ${ }^{\mathrm{TM}}$ system, Oncentra Integrated Prostate Solution device, Elekta-Nucletron, Veenendaal, The Netherlands, a Level II robotic system) [6].

The AAPM and GEC-ESTRO guideline for image-guided robotic BT Report of Task Group 192 issued in October 2014 [1] provides an exhaustive table with all the existing brachytherapy robotic systems. They are analyzed by: (1) RIA class, (2) Level of autonomy, (3) Application, (4) Imaging modality, (5) DOF, (6) Number of channel/needle, (7) Needle insertion, (8) Needle rotation, (9) Angled insertion, (10) Seed delivery, (11) Needle withdraw, (12) Physical template, (13) Template/ perineum area coverage, (14) Depth movement, (15) TPS, (16) Needle-tip positioning accuracy in the air, (17) Needle-tip positioning accuracy in phantom, (18) Accuracy in seed deposition, (19) Emergency stop, (20) Provision for reverting to conventional mode, (21) Force-torque sensor, (22) FDA approval. A brief summary of the current brachytherapy robotic systems, their developers, applications, and imaging modality is given in Figure 1 [1]. The mean calibration error for the prostate BT robots is in the range of $0.5 \mathrm{~mm}$. In tissue mimicking phantoms, the mean needle angulations' error at $10 \mathrm{~cm}$ depth is around $0.5^{\circ}$, translating into a seed placement overall mean error of around $3 \mathrm{~mm}$. In practice, the main contributor for error is the needle deflection through the real prostate (due to different tissue densities including cancer stiffness, adenoma elasticity, prostate calcifications, or previously inserted seeds, etc.).

The majority of systems have thus been tailored for prostate and lung source implantation (the latter to a lesser extent). Therefore, implementing a universal BT robotic system is one of the field's main directions of development $[7,8]$.

\section{Our experience: the Para-BrachyRob (PBR)}

A solution to the above mentioned limitations was proposed by The Research Center for Industrial Robots Simulation and Testing (CESTER) Cluj-Napoca, Romania [9], which developed a family of modular parallel robots for brachytherapy (BR1, BR2, BR3) (Figure 2) for HDR brachytherapy. The aim was to obtain CT-based dosimetry for inoperable cancers with robotic assisted needle 


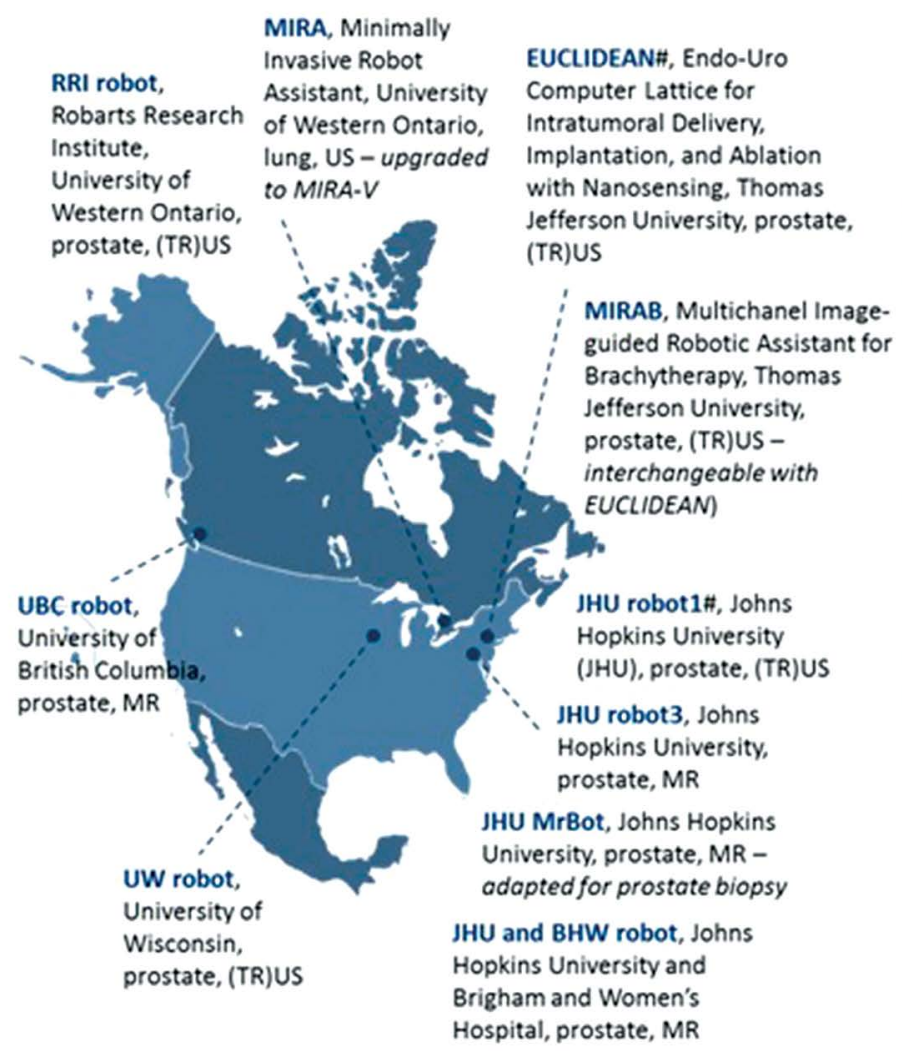

FIRST\#*, Fully Integrated

Real-time Seed Treatment,

Elekta-Nucletron,

prostate, (TR)US

UMCU robot, University

Medical Center Utrecht,

prostate, (TR)US

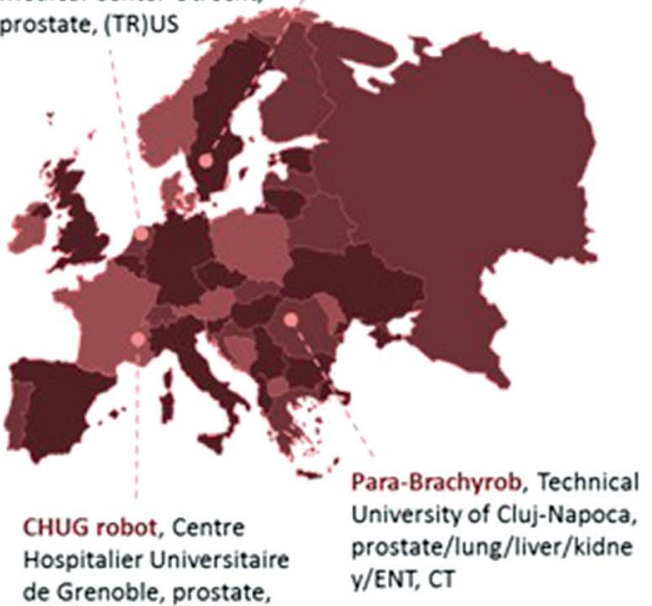

de Grenoble, prostate,

(TR)US

Fig. 1. Picturing the centers currently involved in developing brachytherapy robotic systems. Key: Name, Institution/Company, Targeted organ, Imaging modality; \#the only systems used on patients for clinical brachytherapy; *the only commercially available system (approved by FDA and CE)

placement (thoracic, abdominal, and pelvic cancers) (Figure 3). Based on simulation results and on the expertise of radiation oncology specialists, the last system, BR3, was selected for further development into an experimental model. The constructive solution is a CT-compatible, remote control, and portable system with 5 degrees of freedom (DOF): $\mathrm{X}, \mathrm{Y}, \mathrm{Z}$, angulation and rotation. It is small enough to fit the $80 \mathrm{~cm}$ gantry when mounted on the CT-table but it can be also fixed to the ground. The system has a backup battery.

The kinematic scheme of PBR consists of two cylindrical modules of 3 DOF each. Besides the similar first two active joints, which perform a translational movement, one of the modules associates a third rotational joint. They both associate two distal passive joints, while an additional active joint is characteristic of the needle insertion module. Thanks to this structure, the BR3 model of PBR eliminates the fixed frame of the previous two models, BR1 and BR2 [10].

In brief, the system's technical requirements include: (1) 5-DOF $(X, Y, Z, \Psi, \theta)$, (2) double rigid grip for needles of various lengths $(5-25 \mathrm{~cm})$, (3) sterile cap for needle contact, (4) sensor for resistance and for vibration, with dual signalization (audio and visual), (5) precision of in vivo contact $<3 \mathrm{~mm}$ in any direction, (6) automatic charger for up to 20 needles, and (7) double safety blocking mechanism. We are currently in the pre-clinical phase. The virtual patient is placed in the treatment position on the CT couch. A contiguous $2 \mathrm{~mm}$ slice thickness CT acquisition of the zone of interest is done. As principle, the coordinates of the target are linked with the external tattoos (as in EBRT), the attaching points of the robot to the couch, and with the fiducials placed in the tumor or in its' close proximity. The contouring of the target and organs at risk is done like for a classical EBRT planning. The software proposes solutions for needle placement, avoiding the organs at risk (pre-plan). Optimization is performed by different angles insertions if needed, until all the dosimetry predefined constraints are fulfilled. Final approval by the physician and physicist in charge ends the planning procedure. The next day, the patient, under local, spinal or general anesthesia (as required by the tumor localization) is repositioned the same way on the CT-couch (laser alignment on the tattoos, as for EBRT), and the robot is rigidly attached to the couch in the specially designed articulation points (visible on the CT images). Then the robot automatically moves to the insertion start position, according to the shifts generated by the planning system and starts the implantation, with the most "safe" needle, under CT guidance in any particular moment asked by the operating team. The procedure is checked periodically from outside the room by couples of CT slices (or alternatively from inside the operating room by trans-abdominal ultrasound) and pursuit catheter by catheter, if only minor deviations are encountered. Final CT acquisition is done for the real dosimetry, and HDR (or PDR) BT plan 
A

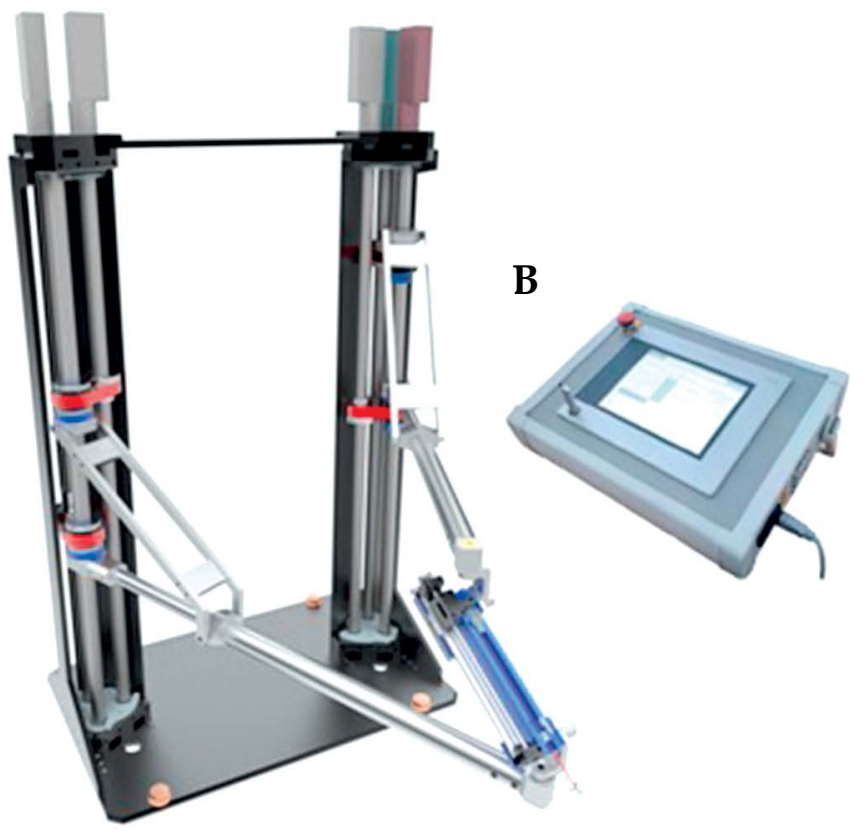

C

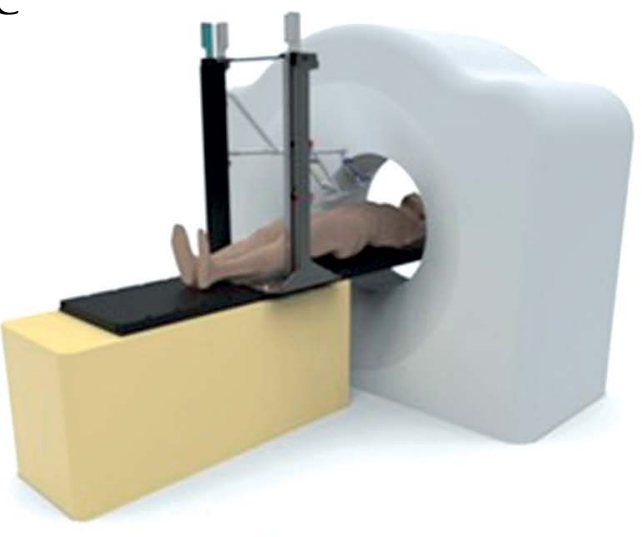

D

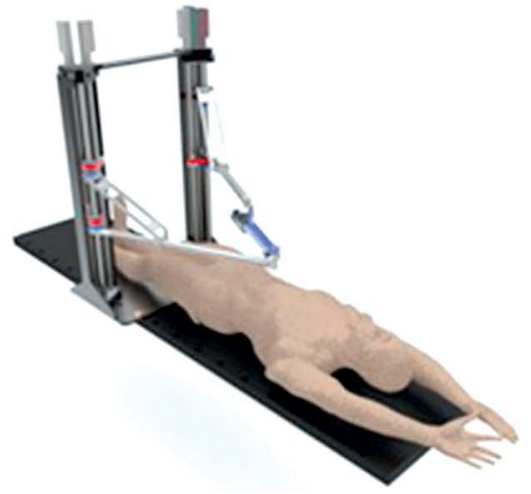

Fig. 2. Para-BrachyRob brachytherapy system components and use: A) cylindrical modules and arms; B) control unit; C) system fixed on the CT-table; D) system fixed on the ground

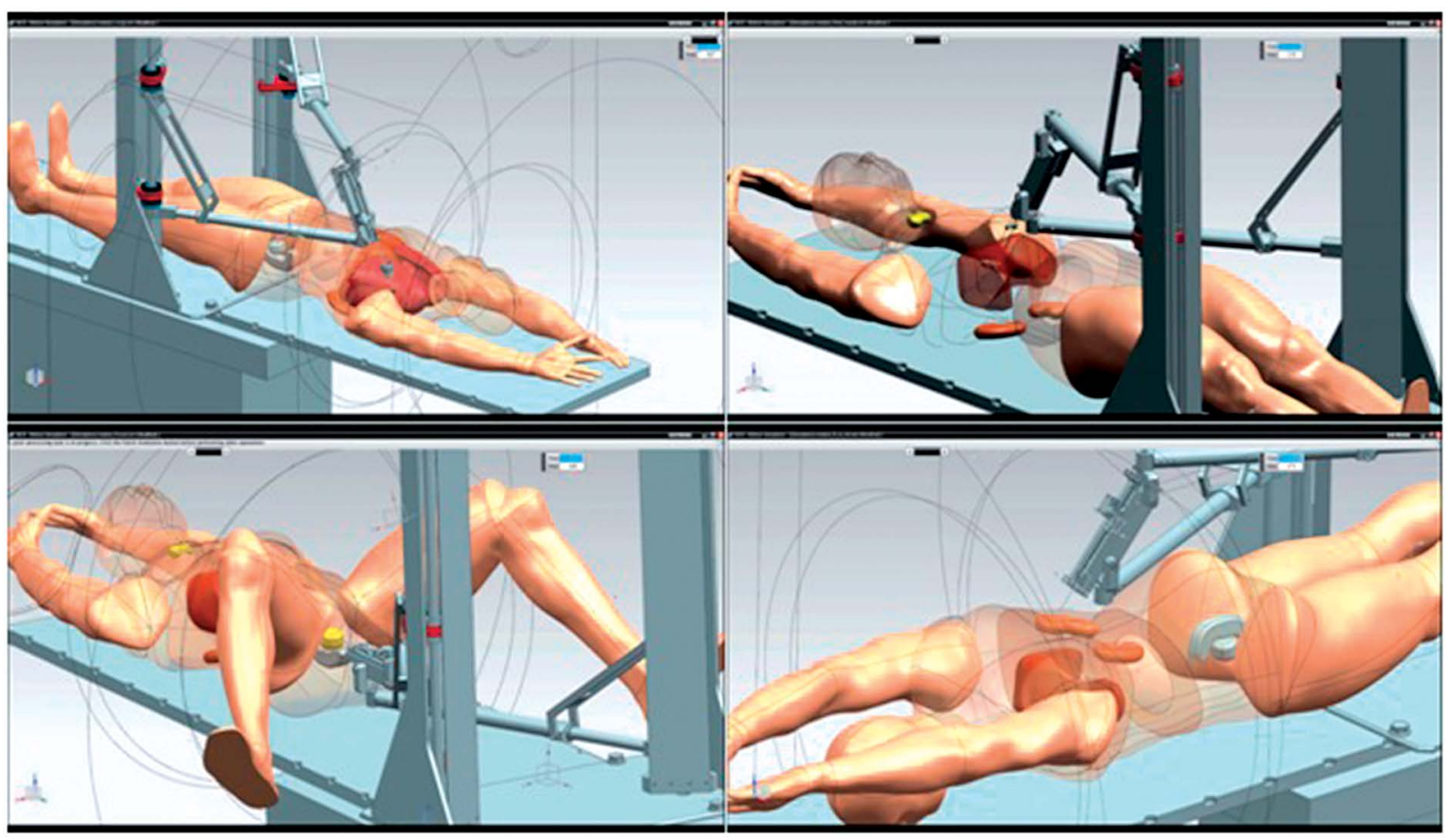

Fig. 3. 3D simulation of Para-BrachyRob robotic assisted brachytherapy with the cylindrical modules mounted on the CT-table (from top left): applications in lung, liver, prostate, and kidney cancers 
can be delivered. PBR can be used also for biopsies and LDR seed placement.

\section{Conclusions}

At present, automate (partial or full procedure) seems to be the best solution for bringing BT back to stage. By increasing its availability and consequently reducing the technique's learning curve, more junior radiation oncologists are expected to turn their attention to BT, and, consequently, more patients could benefit. For the time-being, the Oncentra Integrated Prostate Solution device is the only robotic system commercially available for LDR seeds BT. Among a dozen of custom-made and under development systems, our parallel robot Para-Brachyrob, thanks to its versatility and technical capabilities, might be a pioneer of the next generation precision tools in BT.

\section{Acknowledgements}

This study was kindly supported by the UEFISCDI through project no. 173/2012, code PN-II-PCCA-20113.2-0414, entitled "Robotic assisted brachytherapy, an innovative approach of inoperable cancers - CHANCE", by the Scopes International Grant IZ74Z0_137361/1, "Creative Alliance in Research and Education focused on Medical and Service Robotics-CARE-Robotics" and by the POSDRU program through project DMI 1.5, ID 137516-PARTING.

We are also thankful to Professor Dan Stoianovici, $\mathrm{PhD}$, from the Brady Urological Institute, Johns Hopkins University, Baltimore, US for his logistical support concerning the latest advances in robotic brachytherapy systems at the JHU, as well as to the staff at the Technical University of Cluj-Napoca and at the Research Center for Industrial Robots Simulation and Testing (CESTER), Romania, for providing the technical chart of their custom-made developed robot, Para-BrachyRob. The authors are grateful to Margareta Moga, for her research contribution.

\section{Disclosure}

Authors report no conflict of interest.

\section{References}

1. Podder TK, Beaulieu L, Caldwell B et al. AAPM and GECESTRO guidelines for image-guided robotic brachytherapy: report of Task Group 192. Med Phys 2014; 41: 101501.

2. Sheridan TB. Telerobotics, Automation and Human Supervisory Control. MITPress, Cambridge 1992.

3. Rivard MJ, Evans DA, Kay I. A technical evaluation of the Nucletron FIRST system: conformance of a remote afterloading brachytherapy seed implantation system to manufacturer specifications and AAPM Task Group report recommendations. J Appl Clin Med Phys 2005; 6: 22-50.

4. Podder TK, Buzurovic I, Huang K et al. Reliability of EUCLIDIAN: an autonomous robotic system for image-guided prostate brachytherapy. Med Phys 2011; 38: 96-106.

5. Fichtinger G, Fiene J, Kennedy CW et al. Robotic assistance for ultrasound guided prostate brachytherapy. Med Image Comput Comput Assist Interv 2007; 10 (Pt 1): 119-127.
6. Deborah RK, Stoianovici D, Han M et al. Robotic ultrasound and needle guidance for prostate cancer management: review of the contemporary literature. Curr Opin Urol 2014; 24: 75-80.

7. Borangiu $\mathrm{T}$ (ed.). Advances in Robot Design and Intelligent Control. Springer International Publishing, Switzerland 2014.

8. Jun H, Shin HW, Lee S et al. Biomedical Engineering: Frontier Research and Converging Technologies. Springer International Publishing, Switzerland 2014.

9. Plitea B, Szilaghyi B, Pisla D. Kinematic analysis of a new 5-DOF modular parallel robot for brachytherapy. Robot ComInt Manuf 2015; 3: 70-80.

10. Pisla D, Plitea N, Galdau B. Innovative Approaches Regarding Robots for Brachytherapy in New Trends in Medical and Service Robots. Springer International Publishing, Switzerland 2014. 